\title{
EXPERIENCIA ACADÉMICA Y ESTRATEGIAS DE COMPRENSIÓN LECTORA EN ESTUDIANTES UNIVERSITARIOS DE PRIMER AÑO*
}

\author{
Angie Carolina Neira Martínez ${ }^{* *}$ \\ Fernando Teddy Reyes Reyes ${ }^{* * *}$ \\ Bernardo Esteban Riffo Ocares ${ }^{* * * *}$
}

\begin{abstract}
Resumen
La comprensión lectora es fundamental en el éxito académico universitario, por lo que se espera que el estudiante que ingresa a la educación superior posea competencias lectoras suficientes, lo que, a menudo, no es así. Este estudio tuvo por objetivos establecer, por un lado, el nivel de comprensión lectora con el que ingresa un grupo de estudiantes a una carrera de pedagogía y, por otro, describir las estrategias de lectura que emplea un sub grupo de dichos estudiantes. Para ello, se realizó un estudio mixto de corte transversal de alcance descriptivo, sobre la base de una muestra no probabilística, en el que se aplicaron dos instrumentos: una prueba de comprensión lectora (Lectum 7) y un protocolo de pensamiento en voz alta. Los resultados revelan un desempeño promedio general bajo, con una comparación de lectores hábiles y menos hábiles que arroja una diferencia significativa en cuanto a nivel de comprensión lectora, y una comparación entre estudiantes con y sin estudios superiores previos favorable a los primeros. Además, se observa una diferencia en la eficacia con la que se emplean estrategias de comprensión lectora, que muestra una mayor reflexión por parte de los lectores hábiles. Finalmente, es posible concluir que el empleo de estrategias y la experiencia con distintos tipos de texto son factores que inciden en el desempeño lector.
\end{abstract}

Palabras clave: comprensión del discurso, evaluación de la comprensión lectora, estrategias de lectura.

\section{ACADEMIC EXPERIENCE AND READING COMPREHENSION STRATEGIES IN FREHMEN UNIVERSITY STUDENTS}

\footnotetext{
Abstract

Reading comprehension is crucial in the academic success. When students enter university, it is expected that they have a sufficient level of reading, but this isn't always the case. The objectives of this study are to know the level of reading comprehension of a group of

* Artículo inscrito en la tesis doctoral de la autora principal, titulada La lectura en la educación superior: el papel del texto y del uso de estrategias en la comprensión lectora de estudiantes de primer año.

** Chilena, Doctora (c) en Lingüística, Becaria Conicyt. Universidad de Concepción. Concepción, Chile. angieneira@udec.cl

*** Chileno. Doctor (c) en Metodología. Universidad del Desarrollo. Concepción, Chile. freyes@udd.cl

**** Chileno. Doctor en Lingüística. Universidad de Concepción. Concepción, Chile. bernardo.riffo@ udec.cl
} 
freshmen students of pedagogy, and to recognize reading strategies used by a sub group. This is a transversal and descriptive study, with a non-probabilistic sample. The measures were done with two instruments: a reading comprehension test and a verbal protocol. The results show a low average achievement, a significant difference in achievement between competent readers and less competent readers, and a significant difference in achievement between students with university experience and students that come straight from high school. Furthermore, it is noticeable that competent readers use reading strategies effectively, which shows a larger reflection. In sum, it is possible to conclude that strategy use and academic experience are factors that affect reading achievement.

Keywords: discourse comprehension, reading comprehension assessment, reading strategies.

Recibido: 06-06- 2014

Aceptado: 24-12-2014 


\section{Introducción}

Es innegable la estrecha relación que existe entre lectura y aprendizaje, dado que una de las principales fuentes de conocimiento es el texto escrito. A esto se debe la relevancia que cobra la comprensión lectora en el desarrollo educativo, puesto que ello es fundamental en el éxito académico, lo que se observa desde el comienzo de la vida escolar hasta la educación superior (Marín, 2006).

Cuando un estudiante ingresa a la universidad, se espera que el desarrollo de sus habilidades lectoras haya alcanzado el nivel adecuado para enfrentar los estudios superiores. Sin embargo, y cada vez con mayor frecuencia, las instituciones de educación superior han notado las dificultades de sus alumnos para comprender lo que leen (i.e. Makuc, 2011; Maldonado, Sandoval y Rodríguez, 2012; Sandoval, Frit, Maldonado y Rodríguez, 2010; Velásquez, Cornejo y Roco, 2008). Por ello, no nos sorprende que este tema despierte el interés de diversos investigadores (Calderón-Ibáñez \& Quijano-Peñuelas, 2010; Echeverría y Gastón, 2000; Marciales, 2003; Irrazabal, Saux, Burin y León, 2005; Martínez-Díaz, Díaz y Rodríguez, 2011; Riffo \& Contreras, 2012).

Además de la comprensión en profundidad de los textos de estudio, es imprescindible la conciencia que adquiere el individuo para reconocer los desafíos y dificultades que le presenta el discurso (Cerchiaro, Paba y Sánchez, 2011). Para aprender desde el texto, los procesos de metacognición resultan decisivos, ya que los lectores comprenden y aprenden mejor a partir de un texto escrito cuando logran cierto grado de control sobre el proceso mediante el monitoreo y uso estrategias de lectura (McNamara, 2004a, 2004b, 2010).

Este artículo da cuenta de una investigación en estudiantes de primer año de una carrera universitaria, trabajo en el que se estudia la relación entre la comprensión lectora y la experiencia académica, al mismo tiempo que se describen las estrategias utilizadas por los lectores. En función de lo anterior, es necesario primero establecer fundamentos teóricos que den cuenta del proceso de comprensión lectora, cómo se puede medir la capacidad de los individuos para realizar esta completa tarea y cuáles procedimientos son los más frecuentes en los lectores a la hora de resolver un problema de esta naturaleza. 


\subsection{Comprensión lectora}

La comprensión lectora es un proceso cognitivo complejo, requisito necesario para muchas otras operaciones de alto nivel, como el aprendizaje o el razonamiento, cuando en ello se ve implicado material textual escrito. En el presente estudio, se aborda este fenómeno desde el modelo de construcción-integración de Kintsch (1998), teoría ampliamente aceptada, de gran influencia en la psicolingüística, toda vez que propone una explicación completa del procesamiento cognitivo y de las representaciones mentales involucradas en la comprensión del lenguaje (Vieiro \& Gómez, 2004).

El modelo de construcción-integración explica el fenómeno de la comprensión del discurso a través de la propuesta de una teoría de procesos psicológicos: describe las etapas del procesamiento cuyo resultado es una serie de representaciones mentales elaboradas a partir del texto leído (Kintsch, 1998; Kintsch \& van Dijk, 1978). Dos procesos principales conforman los mecanismos básicos del modelo, de los cuales deriva también su nombre: uno de construcción y otro de integración. En el primero se activa la información del texto y el conocimiento relacionado con él, sea esta relevante o irrelevante. La construcción se produce de abajo hacia arriba y en forma rápida, automática y caótica. En el segundo proceso, se despliega la activación a lo largo de la red de proposiciones hasta que esta se decanta; esto es, se seleccionan los conceptos que se relacionan y se desactivan aquéllos que son irrelevantes (Kintsch, 1998; Kintsch \& Rawson, 2005). Cabe mencionar que la construcción es un proceso de recuperación, mientras la integración es uno de activación (McNamara \& Magliano, 2009).

Como resultado del proceso general de comprensión, el lector elabora, entre otras, tres tipos de representación en la memoria, a saber, de superficie, base de texto y modelo de situación:

a. Representación de superficie. Este es el resultado del procesamiento de palabras y frases. En este nivel están involucradas las habilidades de decodificación, reconocimiento de palabras y análisis sintáctico (parsing).

b. Representación semántico-proposicional o texto base. Este nivel es resultado del análisis de la micro y de la macroestructura, las 
que determinan el significado del texto. La primera está formada por una compleja red de proposiciones interrelacionadas, que psicológicamente se construye de acuerdo a la vinculación sintáctica entre las palabras en el texto y a los nexos de coherencia entre proposiciones. La segunda se forma al reconocer las ideas globales del texto y la interrelación entre ellas. El texto base corresponde al significado de las expresiones que explícitamente se expresan en la superficie del texto.

c. Modelo de situación. Este es un modelo mental de la situación referida por el texto, y es el resultado de la integración entre la información entregada por el texto, los conocimientos previos del lector y sus metas para comprender. Esta representación permite lograr una comprensión profunda del texto. La construcción del modelo de situación no depende solo del dominio verbal, sino que se extiende a imágenes, emociones, experiencias personales, entre otros recursos cognitivos y de memoria.

De los tres niveles de representación, los de mayor impacto en la comprensión del discurso son el texto base y el modelo de situación (Kintsch \& Rawson, 2005).

\subsection{Evaluación de la comprensión lectora}

Evaluar la capacidad de comprensión lectora supone, primero, un marco teórico que explique en qué consiste la lectura comprensiva y, segundo, un conjunto de aspectos a evaluar, con sus correspondientes criterios y parámetros. El modelo de evaluación de la comprensión lectora propuesto por Véliz y Riffo (1993) y Riffo y Véliz (2011), cuya finalidad es guiar la construcción de un instrumento de evaluación que integre los diversos aspectos que participan en el proceso lector, se propone entregar parámetros de medición que permitan clasificar a los lectores de acuerdo con el nivel alcanzado en las distintas dimensiones que implica el complejo proceso de la comprensión de lectura. Su sustento psicolingüístico se encuentra principalmente en los modelos de comprensión vigentes (Kintsch, 1998; Kintsch \& van Dijk, 1978), el que es apoyado por el concepto de género discursivo (Bhatia, 2004, Swales, 1990;). A partir de lo anterior, se considera que el proceso de comprensión lectora implica un lector, un texto y su contexto, los que interactúan de forma dinámica. De esta manera, se puede focalizar la mirada 
por separado en estas interacciones, para identificar así habilidades específicas.

El modelo de evaluación distingue tres dimensiones de la comprensión lectora: a) la comprensión textual, que corresponde a criterios determinados por el nivel de procesamiento requerido por la tarea en función del nivel de la estructura del texto en el que se sitúa la tarea de comprensión; b) la comprensión pragmática, que especifica criterios determinados por la relación del texto con su contexto; y c) la comprensión crítica, correspondiente a la relación entre el lector, el texto y su contexto, destacándose aquí la posición que el lector puede adoptar respecto del texto. Cada una de estas dimensiones incluye varios subaspectos, definidos en función de las operaciones lingüístico-cognitivas que el proceso de comprensión requiere y que a continuación se detallan.

En la dimensión de comprensión textual se mide la habilidad del lector para resolver tareas en distintos niveles de organización, desde la palabra hasta el texto en su conjunto. Esta dimensión incluye tres niveles:

1. Nivel proposicional, en el que se miden dos aspectos: a) la comprensión de palabras poco frecuentes con dos indicadores: recuperar o reconocer significado a través de claves extraídas del texto, donde la información se encuentra explícita, y generar significado a partir de información implícita en el texto; y b) la comprensión de oraciones con dos indicadores: identificar o relacionar predicados y argumentos e identificar circunstancias, en ambos casos la información puede encontrarse explícita o implícita en el texto.

2. Nivel microestructural, en el que se mide la comprensión de secuencias de dos oraciones a partir de tres indicadores: a) establecer relaciones de coherencia referencial, b) establecer relaciones de coherencia condicional (que incluyen relaciones temporales y causales), c) establecer relaciones de coherencia funcional (es decir, comprender cómo operan los organizadores textuales). En los tres casos la información puede estar explícita o implícita en el texto.

3. Nivel macroestructural y superestructural, en el que se mide la comprensión de secuencias de más de dos oraciones, párrafos o el texto en su conjunto a través de cuatro indicadores: a) reconocer o derivar el significado global del texto, b) establecer relaciones de coherencia condicional, c) establecer relaciones de coherencia funcional y 
d) determinar la organización lógica del texto (es decir, realizar operaciones relacionadas con el procesamiento de la superestructura). En los cuatro casos, la información puede encontrarse de manera explícita o implícita en el texto.

En la dimensión de comprensión pragmática, el objeto de evaluación es la habilidad del lector para establecer relaciones entre el texto y su contexto, tanto el inmediato como el cultural. Esta dimensión se vale de cuatro indicadores: a) reconocer los participantes en el círculo comunicativo del texto, esto es, distinguir entre enunciado y enunciación; b) situar el texto en el contexto inmediato y en el contexto cultural; c) determinar los propósitos del texto; y d) establecer qué sentido adquiere el texto, de acuerdo con el contexto en el que circula. En los cuatro aspectos, la información puede encontrarse explícita o implícita en el texto.

En la última dimensión considerada en el modelo, se evalúa la capacidad del lector para relacionarse críticamente con el texto, lo que implica componentes metacognitivos y reflexivos. Esta dimensión es evaluada a partir de cuatro indicadores: a) transferir información, por ejemplo, re-contextualizar información, utilizar la información para resolver problemas y realizar otras actividades de naturaleza reflexiva; b) emitir juicios de valor; c) discutir, es decir, dialogar con el texto; y d) comprender significados no convencionales y operaciones retóricas del discurso (lenguaje figurado).

\subsection{Estrategias de comprensión lectora}

La lectura es un proceso de comunicación interactivo y complejo, compartido por autor y lector. Para reconstruir el mensaje, el lector, cumpliendo un rol activo y participativo, debe saber leer las pistas que dejó el autor. En este proceso comprensivo las estrategias de lectura son fundamentales (Parodi, Peronard \& Ibañez, 2010).

Los procesos de metacognición son esenciales en la utilización de estrategias de lectura, dado que le permiten al lector reconocer que un nivel superficial de comprensión no es suficiente para aprender (Kintsch, 2009). Esto, porque los lectores comprenden y aprenden mejor a partir de un texto escrito cuando monitorean su comprensión y usan estrategias de lectura como, por ejemplo, efectuar una lectura previa, predecir, generar inferencias, aprovechar su conocimiento previo y hacer resúmenes 
(Best, Ozuru \& McNamara, 2004; McNamara, 2004a, 2010 O’Reilly \& McNamara, 2002).

Se puede definir las estrategias de comprensión lectora como aquellos procedimientos cognitivos y lingüísticos que un lector realiza con el fin de cumplir un determinado objetivo cuando enfrenta una tarea de lectura (Parodi et al., 2010). Se trata de aquellas acciones conscientes que se llevan a cabo en una situación de comprensión difícil para el lector, en la que debe acudir a un procesamiento de tipo top-down (McNamara \& Magliano, 2009).

Las estrategias no solo permiten cumplir un objetivo, sino que también se utilizan para re-direccionar o re-organizar un plan de lectura al constatar que este no tiene éxito (Parodi et al., 2010; Peronard, 2002). Es así como las estrategias de comprensión son particularmente necesarias cuando hay problemas, por ejemplo, cuando el conocimiento del lector es insuficiente o el texto tiene algunas ambigüedades (Bereiter \& Bird, 1985).

Se afirma que las estrategias de búsqueda de coherencia y de explicación son los sellos de una buena comprensión. A medida que avanzan en la lectura, los buenos lectores intentan conectar (a través de inferencias puente) las oraciones nuevas que van apareciendo, tanto con el contenido de las partes del texto ya leídas como con su conocimiento previo sobre el tema. En el caso de la auto-explicación, los buenos lectores se inclina más a preguntarse por qué en lugar de dónde, cómo o cuándo (Graesser, 2007). Esta estrategia consiste en que el lector explique un texto a medida que lo va leyendo (McNamara, 2004b). Los resultados obtenidos a partir del uso de esta estrategia se orientan a sostener que quienes son capaces de explicar el texto construyen un modelo mental más rico y completo (McNamara, 2004a, 2009; O’Reilley, Best \& McNamara, 2004).

Algunas de las acciones que realizan los lectores expertos durante la lectura descritas por Pressley y Afflerbach (1995) son: tomar notas, parafrasear parte del texto, predecir contenidos y/o la estructura del texto y verificar dichas predicciones, ajustar las expectativas iniciales de acuerdo con la información nueva, adecuar las metas de lectura de acuerdo con el contenido del texto, buscar palabras clave, inferir los significados de las palabras, inferir los significados connotativos, relacionar conocimientos previos con la información contenida en el texto, generar la idea principal, releer el texto o partes de él, construir interpretaciones, entre otras. 
Finalmente, la lectura de textos académicos requiere de un desempeño intelectual de alta complejidad (Riffo \& Contreras, 2012). Un estudiante exitoso se pregunta por qué y cómo ocurre lo expuesto en un texto escrito, es inquisitivo y crítico de sus propias respuestas, construye explicaciones y aplica esas explicaciones a la resolución de problemas, autorregula su aprendizaje a través de la generación de sus propias metas, las que intenta lograr a través del monitoreo regulación y control de sus pensamientos y acciones (McNamara, 2010).

En el marco de los antecedentes expuestos, los objetivos de esta investigación son, por una parte, conocer el nivel de comprensión lectora con el que ingresan los estudiantes a la carrera Pedagogía en Español de la Universidad de Concepción y, por otra, profundizar en cómo un grupo de dichos estudiantes lee los textos, conocer las estrategias de lectura que emplea y de qué forma responde a las preguntas planteadas. Considerando las dificultades que muchos estudiantes presentan para comprender textos en sus estudios universitarios, este trabajo pretende identificar, sobre la base del nivel de comprensión lectora de un grupo de estudiantes de primer año de pedagogía, cuáles son las estrategias de lectura que emplean los estudiantes y qué diferencia a aquellos que tienen un buen desempeño de aquellos que tienen un bajo desempeño lector.

\section{Método}

\subsection{Diseño}

Se desarrolló un estudio mixto de corte transversal y alcance descriptivo a partir de una muestra no probabilística de estudiantes universitarios de primer año.

\subsection{Participantes}

La muestra estuvo compuesta de 40 estudiantes de primer año de Pedagogía en Español de la Universidad de Concepción. El 70\% de ellos de sexo femenino y el $30 \%$ restante de sexo masculino. La edad promedio fue de 20,53 años ( $D E=3.95)$. En cuanto a los establecimientos de Enseñanza Media de procedencia, el 55\% provenía de establecimientos particulares subvencionados, el 38\% de municipales y el $7 \%$ de particulares pagados. Para el $52 \%$ de la muestra, esta era su primera carrera, mientras que el 
$48 \%$ restante tenía estudios superiores previos en las áreas humanista (42\%), científica (42\%) y tecnológica $(16 \%)$.

Del total de participantes, se seleccionó una sub-muestra de 10 estudiantes, 5 de alto rendimiento y 5 bajo rendimiento en comprensión lectora, respectivamente, en la misma proporción de sexo y estudios previos para aplicarles un protocolo de pensamiento en voz alta.

\subsection{Instrumentos}

Los instrumentos utilizados fueron la prueba de comprensión lectora Lectum 7 y un protocolo de pensamiento en voz alta. La prueba de comprensión lectora Lectum 7, forma A (Riffo et al., 2011), diseñada originalmente para estudiantes de cuarto año de enseñanza media, estaba compuesta por cuatro textos: dos argumentativos, uno expositivo y uno narrativo, con un total de 32 preguntas de selección múltiple que apuntaban a diferentes dimensiones de la comprensión lectora: textual (a nivel de palabra, oración, microestructura y macroestrucutra), pragmática y crítica. Cada ítem correcto se califica con 1 punto, por lo que el puntaje total corresponde a la sumatoria del puntaje.

En el protocolo de pensamiento en voz alta, se pidió a los estudiantes que releyeran dos de los cuatro textos usados en la prueba (texto expositivo y texto argumentativo) y que verbalizaran lo que pensaban mientras leían. Una vez finalizada la lectura, se les hicieron algunas preguntas en torno a los comentarios realizados durante la lectura o a estrategias que hubieran usado. Además, se seleccionaron dos preguntas por texto y se les pidió que reconstruyeran la forma en que las habían contestado.

\subsection{Procedimiento}

La aplicación de la prueba de comprensión lectora se realizó de forma colectiva en sala de clases, previa firma de consentimiento informado por parte de los participantes. Posterior a la aplicación, se seleccionó la sub-muestra de 10 participantes, tanto de alto como bajo rendimiento según el puntaje obtenido. A dicho grupo se le aplicó el protocolo de pensamiento en voz alta, lo que se realizó de forma individual en formato de entrevista, para lo que se le pidió a cada estudiante que re-leyera dos de los cuatro textos que componían la prueba. Durante la lectura, se les indicó que verbalizaran libremente ideas o dificultades que surgieran. 
Una vez terminada la lectura, se les preguntó por las estrategias usadas para resolver los problemas verbalizados, principalmente, asociados a palabras desconocidas y a estrategias de subrayado aplicadas en durante la prueba. Finalmente, se analizaron, con cada estudiante, dos preguntas para cada texto y se le pidió que reconstruyera el proceso efectuado para responderlas.

\section{Resultados}

Los resultados que se presentan describen el rendimiento lector para el total de la muestra $(n=40)$. Adicionalmente, se presentan los resultados cualitativos referidos a las verbalizaciones asociadas a la lectura en relación a las estrategias usadas para leer y resolver las preguntas, lo que se aplica a la sub-muestra $(n=10)$.

\subsection{Resultados cuantitativos}

Se presentan, a continuación, tanto los resultados globales de comprensión lectora como para cada uno de los textos incluidos en la prueba.

Tabla 1. Media y desviación estándar para el rendimiento global y por tipo de textos en la prueba de comprensión lectora $(n=40)$

\begin{tabular}{l|c|l|l|l|l|l}
\multicolumn{1}{c|}{ Tipo texto } & $\begin{array}{c}\text { Cantidad } \\
\text { items }\end{array}$ & \multicolumn{1}{|c|}{$M$} & \multicolumn{1}{|c|}{ DE } & \multicolumn{1}{|c|}{ Min } & Max & $\%$ acierto \\
\hline 1. Argumentativo & 8 & 3.7 & 1.7 & 0 & 7 & 46.3 \\
\hline 2. Expositivo & 10 & 6.0 & 2.0 & 2 & 9 & 59.5 \\
\hline 3. Argumentativo & 6 & 2.5 & 1.3 & 0 & 6 & 40.8 \\
\hline 4. Narrativo & 8 & 4.3 & 1.3 & 1 & 6 & 54.1 \\
\hline 5. Puntaje total & 32 & 16.4 & 3.6 & 10 & 24 & 51.3 \\
\hline
\end{tabular}

Como puede observarse, el rendimiento global promedio es bajo. Se puede apreciar, además, que existe una diferencia de casi $20 \%$ entre el texto que resultó más fácil y el más difícil; incluso en dos de los textos algunos de los participantes tuvieron todas las respuestas equivocadas.

A partir del puntaje global de la prueba, se segmentó la muestra en dos sub-muestras, una de lectores hábiles y otra de lectores menos hábiles. Debido al tamaño de la muestra, se decidió hacer dicha partición 
a base de la mediana estimada sobre el puntaje total. Dado que la variable a contrastar en ambos grupos no distribuía de forma normal, se optó por un contraste no paramétrico. Los resultados se presentan en la tabla siguiente.

Tabla 2. Comparación de medias entre lectores hábiles y menos hábiles en comprensión lectora

\begin{tabular}{|c|c|c|c|c|c|c|}
\hline \multirow[b]{2}{*}{ Tipo texto } & \multicolumn{2}{|c|}{ Rango promedio lector } & \multirow[b]{2}{*}{$\begin{array}{l}\text { U de Mann- } \\
\text { Whitney }\end{array}$} & \multirow[b]{2}{*}{$\begin{array}{c}\text { W de } \\
\text { Wilcoxon }\end{array}$} & \multirow[b]{2}{*}{$\mathrm{z}$} & \multirow[b]{2}{*}{$\mathrm{p}$} \\
\hline & $\begin{array}{l}\text { Hábil } \\
(\mathrm{n}=20)\end{array}$ & $\begin{array}{l}\text { Menos hábil } \\
\quad(\mathrm{n}=20)\end{array}$ & & & & \\
\hline 1. Argumentativo & 25.25 & 15.75 & 105.0 & 315.0 & -2.62 & .009 \\
\hline 2. Expositivo & 28.65 & 12.35 & 37.0 & 247.0 & -4.46 & .001 \\
\hline 3. Argumentativo & 25.18 & 15.83 & 106.5 & 316.5 & -2.62 & .009 \\
\hline 4. Narrativo & 23.53 & 17.48 & 139.5 & 349.5 & -1.71 & .088 \\
\hline 5. Puntaje total & 30.48 & 10.53 & 0.5 & 210.5 & -5.43 & .001 \\
\hline
\end{tabular}

Si se considera el rendimiento de los lectores en toda la prueba, se observa que existen diferencias estadísticamente significativas entre los grupos, diferencias que se explican a partir del desempeño en tres de los cuatro textos incluidos. En el único texto donde no se logra una diferencia estadísticamente significativa en las medias de puntajes es en el texto 4, correspondiente a un texto narrativo.

Del total de la muestra, 18 estudiantes (45\%) tenían experiencia universitaria previa, de estos, $13(65 \%)$ corresponden a lectores hábiles. Por su parte, $15(75 \%)$ de los veinte lectores menos hábiles no tenían estudios superiores previos. De esta forma, para conocer el efecto de dicha condición, se realizó el contraste respectivo, a partir del que se encontraron diferencias estadísticamente significativas en el puntaje total de la prueba y en el texto narrativo (texto 4) en favor de los estudiantes con experiencia académica previa. 
Tabla 3. Comparación de medias entre lectores con y sin estudios superiores previos en comprensión lectora

\begin{tabular}{l|c|c|c|c|c|c} 
& \multicolumn{2}{|c|}{ Rango promedio lector } & \multicolumn{4}{|c}{} \\
\cline { 2 - 7 } & $\begin{array}{c}\text { Con estudios } \\
\text { previos } \\
(\mathrm{n}=18)\end{array}$ & $\begin{array}{c}\text { Sin estudios } \\
\text { previos } \\
(\mathrm{n}=22)\end{array}$ & $\begin{array}{c}\text { U de } \\
\text { Mann- } \\
\text { Whitney }\end{array}$ & $\begin{array}{c}\text { W de } \\
\text { Wilcoxon }\end{array}$ & $\mathrm{z}$ & $\mathrm{p}$ \\
\hline 1. Argumentativo & 23.92 & 17.70 & 136.5 & 389.5 & -1.71 & .088 \\
\hline 2. Expositivo & 21.92 & 19.34 & 172.5 & 425.5 & -.702 & .483 \\
\hline 3. Argumentativo & 21.17 & 19.95 & 186.0 & 439.0 & -.338 & .735 \\
\hline 4. Narrativo & 24.67 & 17.09 & 123.0 & 376.0 & -2.13 & .033 \\
\hline 5. Puntaje total & 24.69 & 17.07 & 122.5 & 375.5 & -2.06 & .039 \\
\hline
\end{tabular}

Para someter a prueba la hipótesis de posible interacción entre las variables tipo de lector (hábil-menos hábil) y con y sin estudios previos, se llevó a cabo un ANOVA factorial de 2×2. El resultado correspondiente a "tipo de lector" es $F(1,36)=81.97$, $p<.001$, por lo que se concluye que existen diferencias estadísticamente significativas entre los grupos de este factor, como ya se había presentado. En el análisis de la variable "estudios previos" no se observa un efecto principal $F(1,36)=.043, p<.837$. Finalmente, el efecto asociado a la interacción no resultó estadísticamente significativo $(F(1,36)=.335, p<.566)$

La prueba de comprensión lectora aplicada permite estimar puntajes por dimensiones, considerando un nivel textual, uno pragmático y otro crítico, lo que se obtiene de la sumatoria de los distintos ítems según la función medida y ya no del texto en particular. En la tabla siguiente se presenta la comparación de medias en estos tres niveles entre lectores hábiles y menos hábiles.

Tabla 4. Comparación de medias entre lectores hábiles y menos hábiles en comprensión lectora según dimensiones textual, pragmática y crítica

\begin{tabular}{l|c|c|c|c|c|c}
\hline & \multicolumn{2}{|l|}{ Rango promedio lector } & \multicolumn{4}{|c|}{} \\
\hline Dimensión & $\begin{array}{c}\text { Hábil } \\
(\mathrm{n}=20)\end{array}$ & $\begin{array}{c}\text { Menos hábil } \\
(\mathrm{n}=20)\end{array}$ & $\begin{array}{c}\text { U de Mann- } \\
\text { Whitney }\end{array}$ & $\begin{array}{c}\mathrm{W} \text { de } \\
\text { Wilcoxon }\end{array}$ & $\mathrm{z}$ & $\mathrm{p}$ \\
\hline 1. Textual & 29.78 & 11.23 & 14.20 & 224.5 & -5.05 & .001 \\
\hline 2. Pragmática & 24.13 & 16.88 & 127.5 & 337.5 & -2.16 & .031 \\
\hline 3. Crítica & 25.60 & 15.40 & 98.0 & 308.0 & -2.86 & .004 \\
\hline
\end{tabular}


Como se aprecia en la tabla anterior, en las tres dimensiones evaluadas existe diferencia estadísticamente significativa en el rendimiento de los lectores hábiles respecto de los menos hábiles. Al cambiar la variable de agrupamiento "tipo de lector" por "estudios previos", se presenta diferencia estadísticamente significativa solo a nivel textual $(z=-.74$, $p<.006$ ) en favor de los estudiantes con estudios previos, aunque no se encuentran diferencias estadísticamente significativas a nivel pragmático ni crítico.

\subsection{Resultados cualitativos}

A continuación, se describen de forma cualitativa distintas estrategias empleadas por lectores hábiles y menos hábiles. El nivel de habilidad se determinó de acuerdo con el rendimiento global en la prueba de comprensión lectora y el rendimiento específico en los dos textos sobre los que se les consultó. Debido al tamaño de la sub-muestra, no es posible determinar diferencias estadísticamente significativas entre los grupos.

Tabla 5. Frecuencia de estrategias de lectura empleada por lectores hábiles y menos hábiles

\begin{tabular}{l|c|c|c}
\multicolumn{1}{c|}{ Estrategia } & Total & $\begin{array}{c}\text { Lectores } \\
\text { Hábiles }\end{array}$ & $\begin{array}{c}\text { Lectores } \\
\text { Menos } \\
\text { Hábiles }\end{array}$ \\
\hline 1.Subrayado & 14 & 9 & 5 \\
\hline 2.Deducción de significado de palabras & 7 & 4 & 3 \\
\hline 3. Reconocimiento de la estructura del texto. & 4 & 4 & 0 \\
\hline 4.Cuestionamiento al texto & 3 & 2 & 1 \\
\hline 5.Relectura & 2 & 2 & 0 \\
\hline 6.Formulación de preguntas & 1 & 1 & 0 \\
\hline
\end{tabular}


Como se puede observar en la tabla anterior, el subrayado es la estrategia más ampliamente usada, tanto por lectores hábiles como por menos hábiles. A su vez, las estrategias de relectura, formulación de preguntas y reconocimiento de estructura del texto fueron exclusivamente usadas por lectores hábiles. Se describen, a continuación, estas estrategias y los comentarios que los lectores hacían cuando las empleaban. Se individualizará a los entrevistados según el número originalmente asignado en la base de datos general, presentando el código alfanumérico al final de la cita que incluye número de entrevistado $(\mathrm{E})$, sexo $(\mathrm{h}=\mathrm{hombre}$, $\mathrm{m}=$ mujer) y el porcentaje de rendimiento global como último valor. Así, E2m85, corresponde al entrevistado 2, mujer, que obtuvo un $85 \%$ de rendimiento.

\section{A. Subrayado}

Tanto los lectores más hábiles como los menos hábiles declaran subrayar lo más relevante en el texto, las ideas principales de este. Sin embargo, se observa que los lectores menos hábiles hablan de que subrayan "lo que les llama la atención", lo que indica que su selección de ideas principales muchas veces se basa en una percepción subjetiva y parcial del texto. En el caso de los lectores más hábiles, algunos de ellos declaran hacer dos lecturas y, en la segunda, editar los subrayados. Esta actividad da cuenta de un mayor análisis del texto y una búsqueda de mayor profundidad en la comprensión.

...muchas veces hay cosas que son importantes que no las subrayo, se me pasan de largo y tengo que leer, volver a leer el texto y vuelvo a subrayar, y como que otras veces lo que subrayé antes no era tan importante. (E18h72)

... después de una segunda lectura generalmente, cuando me da el tiempo, voy marcando con más... cómo decirlo... discriminando más qué es más relevante subrayar o no. (E36h75)

\section{B. Deducción del significado de las palabras}

Es común que aparezca alguna palabra en un texto que es desconocida para el lector. ¿Cómo se resuelve ese obstáculo durante la lectura? Una de las estrategias más usadas es deducir el significado de una palabra 
a partir de su contexto. En el texto expositivo, tanto los lectores hábiles como los menos hábiles usaron esta estrategia, aunque un lector hábil es capaz de establecer más relaciones al interior del texto:

La primera palabra, muescas o incisos, yo la voy asimilando con la imagen que está aquí y más adelante la relación que tienen los otros párrafos para poder ir contextualizando las palabras. (E10m56)

En el caso del texto argumentativo, los lectores menos hábiles declaran que no buscan el significado de una palabra desconocida, obvian el fragmento en el que aparece, simplemente continúan la lectura.

Cuando estoy leyendo en mi casa puedo ir a un diccionario o de repente le pregunto a una compañera mía, pero si me sale algo en la prueba así como que obvio esa parte no más, me la salto. (E39m47)

\section{Cuestionamiento al texto}

Los lectores hábiles cuestionan el texto estableciendo una diferencia entre lo que dice el autor y lo que ellos piensan, sus puntos de vista. Por ejemplo, cuestionan posibles contradicciones en el texto o contraponen la postura ideológica del autor con la propia.

...cuando dice al principio que "Karl Marx manifiesta su perplejidad porque las tragedias de Sófocles seguían conmoviendo, a pesar de ser las sociedades modernas tan fundamentalmente distintas", eso no es así, no somos tan distintos. De hecho, después, cuando el autor dice que "mientras más ahondamos en nuestro corazón, más ahondamos en el corazón de cualquier hombre", entonces yo digo ¿no me está diciendo que somos diferentes, las sociedades, la individualidad es la misma? Entonces iba cuestionando el texto. (E29h66)

En el caso deloslectores menos hábiles, tienden a hacer interpretaciones del texto en las que no diferencian la voz del autor de la visión del lector, sino que las mezclan. No son capaces de separar su propia opinión de lo que plantea el autor.

Lo único en que estoy de acuerdo es que el ser humano desea la perfección. ¿Por qué? Porque son imperfectos, la causa. Y aquí encontré la causa, por qué escriben ficciones, yo pienso que a veces narran, no sé, narrador omnisciente, de saberlo 
todo, porque hay un anhelo en el ser humano de ser así, y si un dios no necesita escribir, obviamente. (E5m44)

\section{Relectura}

Un lector hábil lee el texto más de una vez, en particular, si detecta una dificultad u obstáculo en la comprensión. La relectura es una de las estrategias más usadas por los lectores hábiles, puesto que se encuentran en búsqueda constante de coherencia. Por ejemplo:

“...sí, esa parte no la entendí y lo tuve que leer de nuevo para entenderlo" (E18h72).

\section{E. Formulación de preguntas}

Un lector hábil puede aplicar una estrategia como hacer preguntas al texto, en especial si se trata de un texto cuya comprensión será evaluada. Esto es, utiliza preguntas que le permiten hacer inferencias elaborativas, relacionar el contenido del texto con otra información u otras situaciones. Por ejemplo:

"voy pensando en qué preguntas haría yo si estuviera haciendo la evaluación y voy marcando las partes que yo mismo preguntaría" (E36h75).

\section{F. Reconocimiento de la estructura del texto}

Los lectores hábiles reconocen la relevancia de la estructura textual para la comprensión, por lo que disciernen si un texto está estructurado de manera poco habitual después de haber completado la lectura del primer párrafo, por lo menos ya del segundo recién empecé a darme cuenta de qué era lo que se trataba, porque al no tener un título y parte hablando de algo que no se relaciona directamente con la naturaleza del texto mismo, me pareció una especie de digresión que va apoyando la idea que él quiere transmitir. (E36h75)

En síntesis, es posible observar a partir de las estrategias utilizadas que un lector hábil se diferencia de uno menos hábil por el nivel de profundidad de la comprensión. Mientras un lector hábil demuestra un compromiso con la comprensión del texto, el que se refleja en acciones como la relectura o la formulación de preguntas, un lector menos hábil tiende a leer los textos solo una vez y a obviar los obstáculos de 
comprensión para avanzar en la lectura. Además, un mejor lector es capaz de cuestionar un texto, estableciendo la diferencia entre su propio punto de vista y lo planteado por el autor; mientras un lector menos hábil tiende a mezclar su opinión personal con la voz del autor, lo que lo lleva a interpretaciones erradas.

En cuanto a la forma de responder a las preguntas de comprensión, se pudo observar que tanto los lectores hábiles como los menos hábiles usan la técnica del descarte de alternativas para responder a las preguntas. Además, en las preguntas que fueron respondidas correctamente, los lectores menos hábiles se mostraban poco seguros frente a las respuestas que habían entregado y dejaban ver una necesidad constante de regresar al texto para responder a cada interrogante. Una de las preguntas que presentó un mayor desafío tanto para lectores hábiles como para lectores menos hábiles fue extraída del texto expositivo, "Estructura de la población", en la que debían interpretar información a partir de un gráfico. Finalmente, la pregunta que presentó más equivocaciones, en especial entre los lectores menos hábiles, fue extraída del texto argumentativo, "Discurso de Sábato", y medía la comprensión pragmática, de esta forma, se pedía al lector que determinara el propósito del texto.

\section{Discusión y Conclusiones}

Los resultados obtenidos en este estudio revelan que el nivel de comprensión lectora promedio de los estudiantes evaluados es bajo. Esto puede tener consecuencias en el desempeño académico de los sujetos y, probablemente, también en la calidad de sus aprendizajes. Estos resultados son concordantes con otras mediciones nacionales, como el SIMCE (Agencia de Calidad de la Educación, 2013) e internacionales, como PISA (OECD, 2014), que se realizan en enseñanza media.

En cuanto al efecto de la estructura textual en la comprensión, los resultados obtenidos indican que existen diferencias significativas de desempeño entre lectores hábiles y lectores menos hábiles en la lectura de textos argumentativos y de textos expositivos. Estos, asociados al ámbito académico, están constituidos, en su mayoría, por ideas abstractas y menos relacionadas con la experiencia cotidiana de los estudiantes. Tales propiedades textuales explicarían que el procesamiento que lleva a la comprensión sea más complejo y que el nivel de habilidad lectora 
incida en la profundidad de las representaciones mentales resultantes (McNamara, 2004b). Por otra parte, solo en la lectura del texto narrativo no se observan diferencias significativas entre lectores hábiles y menos hábiles, lo que podría explicarse, también, por la naturaleza de la estructura textual, más habitual en la vida cotidiana.

En términos de niveles de comprensión, los dos tipos de lectores identificados se diferencian en los tres niveles evaluados: textual, pragmático y crítico. Esto indica que los lectores menos aventajados tienen falencias en la comprensión, no solo en la construcción del texto base, es decir, en la relación de las ideas explícitas en el texto, sino también en la del modelo de situación, esto es, en la relación entre el contenido del texto y sus conocimientos previos (Kintsch, 2009; Marciales, 2003).

Los datos indican, asimismo, que la experiencia académica impacta el desarrollo de comprensión lectora de los estudiantes (Riffo \& Contreras, 2012). Al comparar a aquellos que cursaron estudios superiores previos con aquellos que ingresaron directamente desde la Enseñanza Media a la universidad, se observaron diferencias significativas en el desempeño global en la prueba. Sin embargo, en términos de los tipos de texto evaluados, solo en el texto narrativo hubo diferencias significativas, lo que indica que los textos expositivos y argumentativos constituyen un desafío equivalente para todos los estudiantes, aun cuando la experiencia académica sea distinta. Acerca de los niveles de comprensión, se observa una diferencia significativa entre estos dos grupos a nivel textual. Esto permite concluir que los estudiantes con estudios superiores previos han mejorado el uso de estrategias de comprensión, principalmente, para construir un texto base más sólido, pero sin llegar a una comprensión más profunda.

En el análisis del uso de estrategias en los estudiantes evaluados, se observa que tanto lectores hábiles como menos hábiles emplearon dos estrategias en común: "subrayado" y "deducción del significado de las palabras". En el caso de la primera, se trata de una estrategia enseñada ampliamente para mejorar el desempeño escolar, por lo que no es raro que sea usada por la mayoría de los estudiantes, quienes declaran subrayar aquello "que les llama la atención" o "que consideran más importante". En el caso de la segunda, frente al obstáculo que constituyen las palabras desconocidas en la comprensión, los sujetos declaran utilizar el contexto $\mathrm{u}$ otras pistas textuales para deducir su significado. Sin embargo, se 
observa una importante diferencia entre los dos grupos de lectores: los lectores más aventajados se ven beneficiados por el uso de este tipo de estrategias, las que les permiten optimizar su comprensión, mientras los lectores menos competentes usan dichas estrategias ineficazmente, pues parecen no ayudarles a comprender mejor.

Además de las estrategias anteriormente mencionadas, se observaron estrategias de lectura empleadas solo por lectores más competentes, como "reconocimiento de la estructura del texto", "relectura" y "formulación de preguntas". Esto da cuenta de una mayor reflexión en torno a la lectura y una búsqueda consciente de la coherencia, lo que es consignado como uno de los sellos de una buena comprensión (Graesser, 2007; McNamara, 2004a, 2004b, 2009; O'Reilley et al., 2004).

Otro de los datos analizados fue la reflexión de los sujetos acerca de la forma de responder a las preguntas de comprensión. Se observa un predominio de la técnica del descarte al enfrentarse a ítems de selección múltiple, lo que se asocia a estrategias escolares, en especial, en ítems donde existen distractores fácilmente descartables que pudieran llevar a la selección de la alternativa correcta aunque no se conozca el contenido. Además, el hecho de que los lectores hábiles se mostraran más confiados en sus respuestas que los menos hábiles puede estar asociado a que los primeros tienen un mayor entrenamiento o mejor dominio de ciertas destrezas que hacen que confíen en sus capacidades de procesamiento de información en contexto de evaluación.

Finalmente, es preciso tener en cuenta las proyecciones de este estudio. En primer lugar, dado que el tamaño muestral es pequeño para poder generalizar los resultados a una población más amplia de estudiantes universitarios, se proyecta replicar el estudio en una muestra mayor y con estudiantes de distintas carreras y/o de distintas universidades. En segundo lugar, a raíz de que hubo un bajo grado de control en la aplicación del protocolo de pensamiento en voz alta, se proyecta realizar esta medición en un contexto experimental. 


\section{Referencias}

Agencia de Calidad de la Educación. (2013). Informe nacional de resultados SIMCE 2012. Santiago, Chile: Autor.

Bereiter, C. \& Bird, M. (1985). Use of thinking aloud in identification and teaching of reading comprehension strategies. Cognition and instruction, 2 (2), 131-156.

Best, R., Ozuru, Y., \& McNamara, D.S. (2004). Self-Explaining Science Texts: Strategies, Knowledge, and Reading Skill. En Y. Kafai, W. Sandoval, N. Enedi, A. Nixon, \& F. Herrera (Eds.). Proceedings of the 6th international conference on learning sciences. New Jersey, NJ: Erlbaum.

Bhatia, V. (2004). Worlds of written discourse. London, England: Continuum.

Calderón-Ibáñez, A. \& Quijano-Peñuelas, J. (2010). Características de comprensión lectora en estudiantes universitarios. Revista Estudios Socio-Jurídicos, 12 (1), 337-364.

Cerchiaro, E., Paba, C. \& Sánchez, L. (2011). Metacognición y comprensión lectora: una relación posible e intencional. Duazary, 8 (1), 99-111.

Echeverría, M. \& Gastón, I. (2000). Dificultades de comprensión lectora en estudiantes universitarios. Implicaciones en el diseño de programas de intervención. Revista de Psicodidáctica [en línea], 10. [Fecha de consulta: 20 de enero de 2014] Disponible en: <http://www. redalyc.org/ articulo.oa?id=17501006>

Graesser, A. (2007). An introduction to strategic comprehension. En McNamara, D. (Ed.), Reading comprehension strategies. Theories, interventions and Technologies. New York, N.Y.: Lawrence Erlbaum Associates.

Irrazabal, N., Saux, G., Burin, D. \& León, J. (2005). El resumen. Evaluación de la comprensión lectora en estudiantes universitarios. En Anuario de investigaciones de Facultad de Psicología UBA, 13, 51-57. 
Kintsch, W. (1998). Comprehension. A paradigm for cognition. New York, NY: Cambridge.

. (2009). Learning and contructivism. En S. Tobias \& T. Duffy (Ed), Constructivist instruction: Success or failure? (pp. 223-241). New York, NY: Routledge/Taylor \& Francis Group.

Kintsch, W. \& Rawson, K. (2005). Comprehension. En M. J. Snowling, \& Ch. Hulme, (Eds.), Science of reading. A handbook. Oxford, England: Blackwell.

Kintsch, W. \& van Dijk, T. (1978). Toward a model of text comprehension and production. Psychological Review, 85, 363-394.

Makuc, M. (2011). Teorías implícitas sobre comprensión textual y la competencia lectora de estudiantes de primer año de la Universidad de Magallanes. Estudios Pedagógicos, 37 (1), 237-254.

Maldonado, A., Sandoval, P. \& Rodríguez, F. (2012). Comprensión lectora en la formación inicial docente: estudiantes de educación general básica en una universidad del Consejo de Rectores. Revista Folios, 35, 33-47.

Marciales, G. (2003). Pensamiento crítico: diferencias en estudiantes universitarios en el tipo de creencias, estrategias e inferencias en la lectura crítica de textos. (Tesis para optar al grado de Doctor en Psicología Educativa). Universidad Complutense de Madrid, Madrid, España.

Marín, M. (2006). Alfabetización académica temprana. Lectura y Vida, 27 (4), 30-38.

Martínez-Díaz, E., Díaz, N., \& Rodríguez, D. (2011). El andamiaje asistido en procesos de comprensión lectora en universitarios. Educación y Educadores, 14 (3), 531-556.

McNamara, D.S. (2004a). SERT: Self-explanation Reading training. Discourse Processes, 38 (1), 1-30. . (2004b). Aprender del texto: Efectos de la estructura textual y las estrategias del lector. Signos, 37 (55), 19-30. 
. (2009). The importance of teaching reading strategies. Perspectives on Language and Literacy, 35, 34-40

. (2010). Strategies to read and learn: overcoming learning by consumption. Medical Education, 44 (4), 340-346.

McNamara, D.S. \& Magliano, J. (2009). Toward a comprehensive model of comprehension. En B. Ross, (Ed.), The psychology of learning and motivation, Vol. 51. (pp. 297 - 372). Burlington, MA: Academic Press.

OECD. (2014). PISA 2012 results: What students know and can do Students performance in mathematics, reading and science (Volume I, revised edition). PISA: Author.

O'Reilly, T. \& McNamara, D.S. (2002). What's a science student to do? In Proceedings of the twenty fourth annual meeting of the cognitive science society. New Jersey, NJ: Erlbaum.

O'Reilly, T., Best, R. \& McNamara, D.S. (2004) Self explanation reading training: effects for low knowledge readers. In $\mathrm{K}$. Forbus, D. Gentner \& T. Regier (Eds.), Proceedings of the 26th Annual Meeting of the Cognitive Science Society (pp. 1053-1058). Mahwah, NJ: Erlbaum.

Parodi, G., Peronard, M. \& Ibáñez, R. (2010). Saber leer. Valparaíso, Chile: Santillana.

Peronard, M. (2002). Conocimiento de estrategias de lectura y metacomprensión. Onomazein, 7, 95-115.

Pressley, M. \& Afflerbach, P. (1995). Verbal protocols of reading: the nature of constructively responsive reading. New York, NY: Routledge.

Riffo, B. \& Contreras, M. (2012). Experiencia académica y comprensión de textos especializados en estudiantes universitarios de Ciencias Políticas. Estudios Pedagógicos, 38 (2), 201-219.

Riffo, B. \& Véliz, M. (2011). Modelo de evaluación de la comprensión lectora. (Informe de avance proyecto Fondef D08I1179). Universidad de Concepción, Concepción, Chile. 
Riffo, B., Véliz, M., Castro, G., Reyes, F., Figueroa, B., Salazar, O. \& Herrera, M. O. (2011). LECTUM. Prueba de comprensión lectora. (Proyecto Fondef D08i1179). Conicyt, Chile.

Sandoval, P., Frit, M., Maldonado, A. \& Rodríguez, F. (2010). Evaluación de habilidades en matemática y comprensión lectora en estudiantes que ingresan a pedagogía en educación básica: un estudio comparativo en dos universidades del Consejo de Rectores. Educar em Revista, 2, 73-102.

Swales, J. (1990). Genre analysis. English in academic and research settings. New York, NY: Cambridge University Press.

Velásquez, M., Cornejo, C. \& Roco, A. (2008). Evaluación de la competencia lectora en estudiantes de primer año de carreras del área humanista y carreras del área de la salud en tres universidades del consejo de rectores. Estudios Pedagógicos, 34 (1), 123-138.

Véliz, M. \& Riffo, B. (1993). Comprensión textual: Criterios para su evaluación. Revista de Lingüística Teórica y Aplicada, 31, 163-190.

Vieiro, P. \& Gómez, I. (2004). Psicología de la lectura. Madrid, España: Pearson. 